\title{
Eckstaedt, Anita (2019). Sichtbar machen und Bildern Sprache geben. Psychoanalytische Kunstbetrachtungen. Giessen: Psychosozial

\author{
Olaf Knellessen (Zürich)
}

Eine geschlossene und eine offene Tür, nebeneinander gemalt und gleichzeitig durch die Zähler der Wand nachdrücklich getrennt, stehen in einer Spannung zueinander. (Eckstaedt, 2019, S. 59)

Aufder linken Seite des Bildes ist von innen her gerade noch der äußere Rahmen der Eingangstür mit Schloss und Riegeln zu sehen. Dieser ist an seiner Kante vielfach eingefasst worden. (...) Er zeugt vom Leben. Im oberen Teil der Tür lässt sich fensterartigein Teilöffnen. Riegel sind oben, unten und seitlich vorgeschoben, die Tür ist also fest geschlossen. In einem Abstand zum Türrahmen ist im Bild rechts eine zweite Tür ebenfalls mit Kasettenfüllungen sichtbar. Sie ist im Gegensatz zur ersten in einen Innenraum geöffnet. Ein Stück weit gibt sie den Blick in diesen dahinterliegenden Raum, einen Toilettenraum frei. (ebd., S. 59)

Ein faszinierender Blick auf das Bild von Isabel Quintanilla La Puerta, minutiöse, detailreiche Beschreibungen, was wird da eingeschlossen, was ausgeschlossen, auf jeden Fall dieses Licht von irgendwo im offenen Raum, das sich auftut, das Bild öffnet, dann das Grau, zu dem es heisst, dass es keine Farbe sei und eben doch: «Schaut man genau hin, enthält das Grau feine grüne, helle gelbe, beige und braune Töne...» (ebd., S. 59) Ein faszinierender Blick in diesem Bild, bei der Betrachterin. In diesem Schweifen, in diesem Hin und Her der Perspektiven, in diesem Assoziieren beginnen sich die Bilder sichtbar zu machen.

Ganz offensichtlich wurden Türen geöffnet, als eine Gruppe von Frankfurter Psychoanalytikerinnen und Psychoanalytikern sich regelmässig mit Jean-Christophe Ammann trafen, um mit ihm durch das von ihm zu dieser Zeit geleitete Museum für Moderne Kunst (MMK) in Frankfurt zu gehen und sich in einzelne Arbeiten oder Werkgruppen zu vertiefen. Anita Eckstaedt war Teil dieser Gruppe, ihrer Diskussionen und Auseinandersetzungen, und diese bereichernde Erfahrung 
wurde Anlass für das vorliegende Buch, für das sie frühere Arbeiten ihrer schon langandauernden Beschäftigung mit der Kunst wieder aufnahm, umarbeitete und durch Betrachtungen zu Werken von Künstlern aus dem MMK ergänzte, auf die sie im Rahmen dieser Gruppe gestossen ist, und die sie besonders gefesselt haben.

Die Spannung zwischen der geschlossenen und der offenen Türe in $L a$ Puerta verweist - der Blick von Eckstaedt bewegt sich weiter im Bild und auf dem Bild - auf die Strom- und Wasserzähler zwischen den Türen, die Spannung wird zu einer der Zeit, zu einer von ablaufender Zeit. Vielleicht ist da ein Warten im Bild, auf etwas, auf jemanden. So scheint das Bild über sich selbst hinaus zu weisen, über die eigenen Grenzen, die sowohl abschliessen wie auch öffnen, verweist damit auf andere Bilder der Künstlerin, die über wiederkehrende Elemente mit ihm zusammenhängen. «Zu meiner Arbeitsweise, die immer auch einen Entstehungsprozess der Bilder einbezieht, gehört die Betrachtung der zuvor, gleichzeitig oder auch später gearbeiteten Werke. Dabei nutze ich das bisher entwickelte Verständnis einerseits zur Stützung meiner Hypothese und führe es andererseits unter der Hinzuziehung von Aussagen anderer weiter.» (ebd., S. 65)

Verhüllte Objekte im Innen und im Aussen, zugedeckte Möbel zeichnen wie das Ablaufen der Zeit eine starke und eindringliche Präsenz von Abwesendem, so werden die Tücher zu Türen zu Vorhängen zu Öffnungen im Haus und um das Haus. Exterieurs und Interieurs wechseln sich ab wie zwei Seiten derselben Münze, spiegeln sich gegenseitig. Und immer wieder die Türen auf diesen Bildern - mal geschlossen, mal geöffnet.

Der Tanz der Bilder kommt mit Eckstaedt bei einem an: La Puerta roja. Diese Tür ist nicht nur rot, es ist die Haustüre und steht weit offen, zeigt das Draussen im Dämmerlicht - und lässt natürlich offen, ob es eine des Morgens oder eine des Abends ist. Eine Twilight-Zone, könnte man beinahe sagen. Es ist als ob die Spannung, das Warten, nun zu einem Ende, zu einer Auflösung gekommen wäre.

Was sich in der Spannung der verschiedenen Türen und Durchgänge, im Wechsel, im Vor und Zurück zwischen den Bildern bis zu der Puerta roja entfaltet und aufspannt, ist für Eckstaedt eine Geschichte. Eine spezielle und konkrete Geschichte, nämlich die vom nicht aufklärbaren Verlust des Vaters der Künstlerin. «Aus der Biografie der Künstlerin (...) ist bekannt, dass der Vater während der letzten Tage des Spanischen Bürgerkrieges von Francofaschisten gefangengenommen wurde, wobei angenommen werden kann, dass er als Widerstandskämpfer gefoltert und ermordet wurde.» (ebd., S. 70) Das geschah in der frühen Kindheit der Künstlerin. 
Mit ihrem sehr aufmerksamen und sehr offenen Blick auf die Bilder erzählt Eckstaedt die Geschichte dieses Verlusts, dieses Schmerzes, des Wartens und einer Trauer, die ihr mit dem Bild von der roten Türe abgeschlossen erscheint. Es war auch diese Geschichte, die die Autorin an den Zeichnungen und Ölbildern von Isabel Quintanilla interessierte. «Sie bewegten mich sehr. Nachdem ich aus ihrer Biografie von einem tragischen Ereignis in ihrer Kindheit erfahren hatte, dem nicht aufklärbaren Verlust des Vaters, begann ich, ihre Bilder unter dem Aspekt der Trauer und ihrer Verarbeitung zu sehen.» (ebd., S. 53)

Darin macht sich der psychoanalytische Zugang in diesen Betrachtungen zur Kunst kenntlich, die über Quintanilla hinaus Werke von Siegfried Klapper, Heinrich Hoffmann - seinen Struwwelpeter -, Stefan Ecker, Andreas Slominski, Andy Warhol, Roni Horn und HM Erhardt umfassen. Auf diese «Weise werden Themen wie «Trauma`, «Empathie und Empathieverweigerung`, «Generationenkonflikt und Generationenablösung〉, 〈List und Intrige〉, ‘Wahrnehmung〉 und abschließend «Landschaft und Vergänglichkeit` bearbeitet. Allen behandelten Werken ist das Thema der «Bewältigung des Lebens mit seinen Unabänderlichkeiten` gemein. Damit ist die Frage nach der jeweiligen Identität eng verbunden.» (ebd., S. 13) Und diese Ausrichtung des psychoanalytischen Zugangs entsteht nicht erst im Betrachten der Werke, geht ihm vielmehr voraus. So heisst es beispielsweise zu Bildern von Siegfried Klappner: «Als ich das sehen konnte, fühlte ich mich geradezu gezwungen, über seine Bilder zu schreiben, um darauf zu verweisen, wie Traumatisierungen ein Leben beherrschen können.» (ebd., S. 21)

So faszinierend und fesselnd - bei den Arbeiten von Andreas Slominski geht es um Fallen, in denen man gefangen und in gewisser Weise auch gefesselt wird - der schweifende Blick auf die Elemente und Aspekte der Bilder, das Netz der Verweise und Assoziationen zwischen ihnen und den Arbeiten auch ist - die Parallele zur gleichschwebenden Aufmerksamkeit der Psychoanalytikerin wird hier durchaus zu Recht gezogen -, so stellt sich dennoch die Frage, wie es mit den Geschichten ist, die dazu erzählt werden. Sie machen den Eindruck, als ob sie schon gewusste Geschichten sind, die man im Bild dann wiederfindet und mit ihm verbindet. Mit dem Titel des Buches Sichtbar machen und Bildern eine Sprache geben, wäre die Sprache dann die der Psychoanalyse und mit ihr würde etwas sichtbar gemacht, was sonst nicht zu sehen ist. "Kunst ist ein Medium der Erkenntnis. So wird mit diesen Bildinterpretationen aus meiner Sicht aufgezeigt, was Psychoanalyse vermag.» (ebd., S. 14)

Ist also solches «Wissen» der Psychoanalyse, die Sprache, die den Bildern gegeben wird, um das von ihnen Gezeigte sichtbar zu machen? Ist der Titel des 
Buches so zu verstehen? Ist die Psychoanalyse die eigentliche Sprache, sind die Geschichten, die sie spinnt und erzählt, die eigentlichen Geschichten? Dieser Eindruck stellt sich bei der Lektüre des Buches zunehmend ein.

Damit wäre das Verhältnis von Kunst und Psychoanalyse mit einem Jargon der Eigentlichkeit, der glaubt auf ein gegebenes Wissen rekurrieren zu können, in Gefahr, etwas einseitig zu werden. Demgegenüber könnte man sich fragen, ob die Bilder - wie nicht zuletzt die Kunst - der Psychoanalyse nicht auch etwas geben könnten, ob man ihrem Spiel von Verweisen und Bezügen, ihrem Spiel mit den Assoziationen nicht mehr trauen könnte? Beispielsweise sind die Fallen von Andreas Slominski - meist Tierfallen - in der Tat solche, in denen man sich verfangen kann. Nicht zuletzt deswegen, weil sie in ihrer exakten und präzisen Nachbildung auf die Frage nach der Kopie verweisen, das Verhältnis von Original und Fälschung so vorführen, dass diese Unterscheidung nicht so eindeutig ist, und vielleicht sogar darauf, wie Orson Welles in seinem Film Ffor Fake mal fragen liess, ob denn die Fälschung nicht viel schöner sein könne als das Original?

Und wenn Andy Warhol in seinem «Historienbild» Daily News nur die Front- und die Rückseite dieser immens dicken Ausgabe malte, hat er mit den Schlagzeilen dieser Seiten sicher auch auf das Verhältnis von Gewinnen und Verlieren angespielt, aber vielleicht zudem kenntlich gemacht, dass das Innere dieser Zeitung, ihr Inhalt, ihm nicht so relevant, ihm nicht als das Eigentliche erscheint, das sich, wenn schon, in dieser Umhüllung, die ja auch eine Umarmung ist, erst ergibt, von ihr viel eher bestimmt wird.

Kunst-das wird in diesem Buch sehr schön und immer wieder eindrücklich vorgeführt - ist ein Spiel der Formen und Formate, die wiederum nicht einfach nur Gefässe für vorgegebene und schon gewusste Inhalte sind, sondern solchen Inhalt nicht nur ständig wandeln, ihn darüber hinaus in neuen und anderen Formationen und Medien erst generieren. Und so könnte sie, die Kunst, die Psychoanalyse durchaus anregen, dieses in Anschlag gebrachte Wissen und seine schon feststehenden Inhalte zu überdenken, sich von ihren Bildern etwas geben und sichtbar machen lassen, was sie selbst noch nicht gesehen hat.

Wenn also das Licht in den Räumen von Isabel Quintanilla diese Bilder mit allen Helligkeitsgraden erfüllt, dann ist es einfach da, ohne dass man sagen könnte, woher es kommt. Ganz immateriell ist es nicht nur präsent, sondern prägend, es schafft nicht nur das Bild, es malt es nicht nur, es ist das Bild selbst und erzählt die Geschichte des verlorenen Vaters vielleicht weiter: Von dort, wo es herkommt, kommt er, auch dann, wenn er nicht mehr kommen wird. 